\title{
Improvement of the functioning and efficiency of a Code Blue system after training in a children's hospital in China
}

\author{
Yu Shi ${ }^{1}$, Gongbao Liu ${ }^{1}$, Di Cao ${ }^{1}$, Guoping $\mathrm{Lu}^{2}$, Lin Yuan ${ }^{3}$, Yuping Qian ${ }^{3}, \mathrm{Jie} \mathrm{Xu}^{3}, \mathrm{Chengjun} \mathrm{Sun}^{4}$, \\ Mengmeng $\mathrm{Ge}^{5}$, Lingyu $\mathrm{Lai}^{6}$, Xuan Wang ${ }^{7}$, Yiqun Lu ${ }^{8}$, Guoying Huang ${ }^{9}$, Xiaowen Zhai ${ }^{10}$
}

${ }^{1}$ Division of Medical Administration, National Children's Medical Center Children's Hospital of Fudan University, Shanghai, China; ${ }^{2}$ Intensive Care Unit, National Children's Medical Center Children's Hospital of Fudan University, Shanghai, China; ${ }^{3}$ President's Office, National Children's Medical Center Children's Hospital of Fudan University, Shanghai, China; ${ }^{4}$ Department of Endocrinology and Inborn Metabolic Diseases, National Children's Medical Center Children's Hospital of Fudan University, Shanghai, China; ${ }^{5}$ Department of Neonatology, National Children's Medical Center Children's Hospital of Fudan University, Shanghai, China; ${ }^{6}$ Department of General Medicine, National Children's Medical Center Children's Hospital of Fudan University, Shanghai, China; ${ }^{7}$ Department of Anesthesiology, National Children's Medical Center Children's Hospital of Fudan University, Shanghai, China; ${ }^{8}$ Department of Urology Surgery, National Children's Medical Center Children's Hospital of Fudan University, Shanghai, China; ${ }^{9}$ Cardiovascular Center, National Children's Medical Center Children's Hospital of Fudan University, Shanghai, China; ${ }^{10}$ Department of Hematology/Oncology, National Children's Medical Center Children's Hospital of Fudan University, Shanghai, China

Contributions: (I) Conception and design: G Huang, X Zhai; (II) Administrative support: G Huang, X Zhai; (III) Provision of study materials or patients: G Lu, L Yuan, Y Qian, J Xu, C Sun, M Ge, L Lai, X Wang, Y Lu; (IV) Collection and assembly of data: Y Shi, G Liu, D Cao; (V) Data analysis and interpretation: Y Shi, G Liu, D Cao, X Zhai; (VI) Manuscript writing: All authors; (VII) Final approval of manuscript: All authors.

Correspondence to: Xiaowen Zhai. Department of Hematology/Oncology, Children's Hospital of Fudan University, Shanghai 201102, China.

Email: zhaixiaowendy@163.com.

Background: Code Blue is a popular hospital emergency code that is used to alert the emergency response team to any medical emergency requiring critical care. By retrospectively studying Code Blue cases in a children's hospital, we looked for high-risk factors associated with survival and how to improve the effectiveness of Code Blue systems through training.

Methods: Data were collected on age, gender, department, diagnosis, time of Code Blue call activation, time between call and arrival of the Code Blue team, treatment details and outcome before and after the training process from January 2016 to December 2019. Chi-square test and logistic regression analysis were used to analyze the data.

Results: A total of 139 Code Blue cases from the period of January 2016 to December 2019 were retrospectively studied. The wards where Code Blues occurred most frequently were the infectious diseases ward $(n=31,22.3 \%)$, the hematology and oncology ward $(n=30,21.6 \%)$, and the cardiology ward $(n=15$, $10.8 \%$ ). Age, inpatient status, time of arrival, the time of cardiopulmonary resuscitation (CPR), and the cause of shock were all risk factors for death. After the training, the arrival time and recovery time were significantly reduced $(\mathrm{P}<0.01)$. The proportion of patients who were transferred to the ICU had increased $(\mathrm{P}<0.05)$, and the proportion of deaths had decreased $(\mathrm{P}<0.01)$. The survival curve improved $(\mathrm{P}<0.05)$.

Conclusions: It is very important to summarize the risk factors related to Code Blue. It is clear that the efficacy of the Code Blue events improved after training of the hospital staff in the Children's Hospital.

Keywords: Code blue; children's hospital; survival curve

Submitted Jul 02, 2020. Accepted for publication Dec 16, 2020.

doi: $10.21037 / \mathrm{tp}-20-171$

View this article at: http://dx.doi.org/10.21037/tp-20-171 


\section{Introduction}

Code Blue systems are emergency call and management systems for rapid response in hospitals. A Code Blue can be activated by any healthcare personnel for an individual requiring prompt emergency management (1). Once a Code Blue is activated, a medical team with qualifications of advanced life support (ALS) will respond immediately to reach the locality and initiate resuscitation (1). The term "Code Blue" was first used in the Bethany Medical Center in the State of Kansas in the early 1990s (2). The aim of Code Blue is to ensure that trained personnel are deployed to the patient in the shortest possible time $(3,4)$.

The incidence of in-hospital cardio-respiratory arrest has been estimated to be 1-5 events per 1,000 annual hospital admissions, but the rate of survival of these patients to hospital discharge was $0 \%$ to $42 \%$ with the most common range being between $15 \%$ and $20 \%(5,6)$. Although the survival rate of medical emergencies requiring critical care is dependent on the patient's characteristics and underlying disease (7), this rate can be significantly improved by earlier interventions in terms of cardiopulmonary resuscitation (CPR), defibrillation, and advanced life support (8). The aim of this study is mainly to explore the risk factors of the Code Blue and to improve these factors through team training. The purpose of training is to improve the first aid ability of the team and to further improve the survival rate of the Code Blue. Since 2018, we initiated the Code Blue system in our hospital (Children's Hospital of Fudan University) with a training process aiming at an improved survival rate for patients who undergo the Code Blue process. We present the following article in accordance with the STROBE reporting checklist (available at http://dx.doi. org/10.21037/tp-20-171).

\section{Methods}

This is a retrospective clinical intervention study. We reviewed all the patients who received resuscitation after the Code Blue was activated since January 2012. Inclusion criteria: a series of emergencies such as loss of consciousness, convulsions, decreased heart rate, and respiratory arrest, etc., all meet the Code Blue call standards. Exclusion criteria: children with end-stage diseases, parents giving up treatment and other similar factors. At the same time, we compared the death and survival of patients before and after the ALS and PALS life support courses in our hospital. This study passed the ethical review of the unit ethics committee
(No. [2020]460) and followed the Helsinki Declaration (as revised in 2013). Because of the retrospective nature of the study, the requirement for informed consent was waived.

A "Code Blue" is defined as any unexpected medical emergency requiring critical care and activation of a hospital-wide alert. The Code Blue system was implemented in our hospital starting in January 2012. Our hospital is a tertiary hospital that sees an average of 220,000 out-patients and 3,750 in-patients per month. The Code Blue system is initiated by a 24-hour centralized alarmdistributing process that is composed of a 30 -second indoor announcement over the hospital intercom, identifying the patient's location immediately after the call is received (broadcast all over the hospital except operation rooms and intensive care units). Subsequently, sequential phone calls (20 seconds each) are made to working cellphones until the following Code Blue team personnel can be confirmed: a senior resident, an emergency nurse, a senior emergency nurse, an attending physician, and an attending physician in the intensive care unit.

The Code Blue training course, as a core measure to improve the quality of the Code Blue project, has been implemented in our hospital since 2018 (Table 1). According to the 2015 American Herat Association (AHA), our training course included the BLS training course, the PALS training course and the NRP training course. The BLS training course included the written test, skill station test of 1-person and 2-person PPV, CPR, and AED. The PALS training course included the written test, simulation of resuscitation of a cardiopulmonary failure case and an arrhythmia case as a group leader, a skill station test of assisted ventilation, IO and defibrillator. The NRP training course included the written test, simulation of resuscitation of an inactive newborn, a skill station test of assisted ventilation, CPR and epinephrine usage. These courses were retested every 2 years (9). In each analysis cycle, a logistic regression was performed to identify the association between data variables and survival outcome and to guide the improvements. January 2016 to December 2017 was the early stage before training.

\section{Data collection}

All the data came from the health information system. We collected clinical information and prognostic outcomes of children after Code Blue intervention. These materials included: age, gender, diagnosis, time of Code Blue call activation, time between the call and the arrival of the Code 
Table 1 Modified pediatric life support training course

\begin{tabular}{ll}
\hline Course & \multicolumn{1}{c}{ Content } \\
\hline BLS training course & Written test; skill station test of 1-person and 2-persons PPV, CPR, AED \\
PALS training course & $\begin{array}{l}\text { Written test; simulation of resuscitation of a cardiopulmonary failure case and an arrhythmia case as a group } \\
\text { leader; skill station test of assisted ventilation, IO and defibrillator }\end{array}$ \\
NRP training course & $\begin{array}{l}\text { Written test; simulation of resuscitation of an inactive newborn; skill station test of assisted ventilation, CRP } \\
\text { and epinephrine usage }\end{array}$ \\
\hline
\end{tabular}

*, re-test every 2 years. CPR, cardiopulmonary resuscitation; AED, automated external defibrillator; PPV, persistant positive ventilation.

Blue team, treatment details and outcome.

The main outcome was mortality rate. The mortality cases were the cases that died during hospitalization after the Code Blue. The death rate was the mortality cases divided by the total number of Code Blue cases.

\section{Statistical analysis}

SPSS 22.0 was used for statistical analysis. Continuous variables are directly expressed as median and range. Categorical variables are expressed as number (\%). Univariate analysis was conducted using $\chi^{2}$ tests or Fisher's exact test for categorical data. The main etiology and factors leading to Code Blue activation were analyzed by logistic regression. $\mathrm{P}$ values $<0.05$ were considered to indicate statistical significance.

\section{Results}

From January 2016 to December 2019, a total of 139 Code Blue cases occurred. Among them were mainly inpatients [119 cases (85.6\%)]; 108 cases occurred in wards, 27 cases occurred in diagnostic departments and four cases occurred in Outpatient clinic. Among the cases, 53 (38.1\%) died. The departments in which Code Blue calls most frequently occurred were the infectious diseases ward $(22.3 \%)$, the hematology and oncology ward $(21.6 \%)$, the cardiology ward (10.8\%), the neurology ward (7.9\%), and the respiratory ward (7.2\%) (Table 2). The top ten basic diseases included influenza, stem cell transplantation, severe pneumonia, epilepsy, and hepatic insufficiency.

A total of 96 people passed the training course and entered the actual conduction of the Code Blue. A total of 71 Code Blue cases were retrospectively studied for the period of January 2016 to December 2017, the early stage before training. These patients were divided into a death group and a survival group. The mean age of the death group (median 29 months, 1-192 months) was significantly younger than that of the survival group (median 24 months, $1-182$ months) $(\mathrm{P}<0.05)$. The death group had a higher proportion of surgical patients than medical patients $(65.7 \%$, $\mathrm{P}<0.05)$. More cases occurred in the diagnostic department in the survival group $(30.6 \%, \mathrm{P}<0.05)$. The most common activation recorded was shock, with $48.6 \%(n=17)$ in the death group, and it was found to be a risk factor for death by logistic analysis $(\mathrm{P}=0.014)$ (Table 3). The mortality rates of Code Blues caused by dyspnea were $22.8 \%$ before training and $22.2 \%$ after training; those caused by shock were $48.6 \%$ before training and $50.0 \%$ after training; those caused by convulsions was $2.9 \%$ before training and $0 \%$ after training; and those caused by arrhythmia were $25.7 \%$ before training and $27.8 \%$ after training (Table 4 ). All cases in the death group had CPR and were transferred to the ICU, and the CPR time was longer than that in the survival group. Whether the Code Blue occurred during working hours was not significantly different between the two groups. The time of arrival had a $\mathrm{P}<0.05$, indicating a statistically significant effect on survival (Table 5). Through logistic analysis, we found that age, inpatient status, time of arrival, the time of CPR, and the cause of shock were all risk factors for death (Table 3).

By analyzing the survival factors of the Code Blue, we improved it through training. We paid attention to the early recognition of shock in all the patients, especially the inpatients. We trained the team to improve their first aid abilities. After the improvement, we collected the information on 68 cases. There was no statistically significant difference in survival between males and females, age and area. The proportion of Code Blues in surgical departments increased $(\mathrm{P}<0.01)$. Arrival time and recovery time were significantly reduced. The proportion of patients shifted to the ICU has increased and the proportion of deaths has decreased (Table 4). Thus, the survival curve after training was improved $(\mathrm{P}<0.05)$ (Figure 1). 
Table 2 Basic information of Code Blue

\begin{tabular}{|c|c|c|}
\hline & Number & Percentage \\
\hline \multicolumn{3}{|l|}{ Types of subjects } \\
\hline Inpatient & 119 & $85.6 \%$ \\
\hline Outpatient & 20 & $14.4 \%$ \\
\hline \multicolumn{3}{|l|}{ Outcome } \\
\hline Death & 53 & $38.1 \%$ \\
\hline \multicolumn{3}{|l|}{ Place of occurrence } \\
\hline Outpatient clinic & 4 & $2.9 \%$ \\
\hline Diagnostic department & 27 & $19.4 \%$ \\
\hline Ward & 108 & $77.7 \%$ \\
\hline \multicolumn{3}{|l|}{ Department } \\
\hline Infectious diseases ward & 31 & $22.3 \%$ \\
\hline Hematology and oncology ward & 30 & $21.6 \%$ \\
\hline Cardiology ward & 15 & $10.8 \%$ \\
\hline Neurology ward & 11 & $7.9 \%$ \\
\hline Respiratory ward & 10 & $7.2 \%$ \\
\hline Gastroenterology ward & 9 & $6.5 \%$ \\
\hline Neurosurgery ward & 6 & $4.3 \%$ \\
\hline Nephrology ward & 6 & $4.3 \%$ \\
\hline $\begin{array}{l}\text { Rheumatology and immunology } \\
\text { ward }\end{array}$ & 6 & $4.3 \%$ \\
\hline Neonatal surgery & 5 & $3.6 \%$ \\
\hline General surgery ward & 4 & $2.9 \%$ \\
\hline Otolaryngology ward & 3 & $2.2 \%$ \\
\hline Endocrinology ward & 2 & $1.4 \%$ \\
\hline Orthopedics ward & 1 & $0.7 \%$ \\
\hline \multicolumn{3}{|l|}{ Top 10 basic diseases } \\
\hline Influenza & 19 & $13.7 \%$ \\
\hline After stem cell transplantation & 19 & $13.7 \%$ \\
\hline Severe pneumonia & 8 & $5.8 \%$ \\
\hline Epilepsy & 8 & $5.8 \%$ \\
\hline Hepatic insufficiency & 7 & $5.0 \%$ \\
\hline Nephrotic syndrome & 6 & $4.3 \%$ \\
\hline Brain tumor & 6 & $4.3 \%$ \\
\hline Encephalitis & 5 & $3.6 \%$ \\
\hline Dilated cardiomyopathy & 5 & $3.6 \%$ \\
\hline Leukemia & 5 & $3.6 \%$ \\
\hline
\end{tabular}

\section{Discussion}

We conducted a retrospective case analysis and showed that the time before the Code Blue team reached the patients was inversely associated with survival. Through training, the decreased time of arrival of the Code Blue team significantly improved the survival of patients with unexpected critical illness for whom Code Blue calls were activated.

There have been few studies on the application of Code Blue in children. A few studies found that there are many reasons for Code Blues, such as conversive disorder $(26 \%$ to $13 \%)$, syncope $(21.5 \%$ to $19.6 \%)$, convulsion ( $17 \%$ to $13.7 \%$ ), hypoglycemia ( $4.5 \%$ to $3.9 \%$ ), anxiety ( $4.5 \%$ to $1.9 \%)$, and cardiac arrest (1.1\% to $0 \%)$ (2). Cardiac arrest was the main activation criterion, comprising $54.6 \%$ cases of Code Blues in adults(10).Compared with adults, cardiac arrest was not the main reason for Code Blues. The wards where Code Blues occurred most frequently in our study were the infectious diseases ward $(22.3 \%)$, the hematology and oncology ward (21.6\%), the cardiology ward (10.8\%), the neurology ward $(7.9 \%)$, and the respiratory ward $(7.2 \%)$. The top ten basic diseases were influenza, stem cell transplantation, pneumonia, epilepsy, and hepatic insufficiency, among others. In addition to stem cell transplantation, epilepsy, severe pneumonia and hepatic insufficiency, influenza was the first cause of a Code Blue. This finding reminds us that during the flu season, we should evaluate the conditions of children with influenza. In adults, the majority of Code Blue activation patients were male (59\%) and aged between 70 and 89 years of age (43\%) (10). In children, we found that age was a risk factor for death, with younger age having a greater risk.

The purpose of the Code Blue is to improve the success of CPR, and thereby improve the patient survival rate. The Code Blue includes the establishment of a professional team, maintaining the alertness of the team, technological call systems, preparation until the team arrives, the time to arrival of the Code Blue team, availability of equipment, and effective intervention and post-intervention management and records (11). Our training courses used a series of modularized, standardized, and practical teaching courses combined with theoretical learning, case teaching, skill training, and comprehensive assessment so that medical staff can accurately grasp the first aid theory and achieve improved resuscitation. This course adopts the BLS and PALS training courses of the AHA (9), uses the latest version of standardized teaching materials, and uses videoassisted professors to provide students with professional 
Table 3 Analysis of all variables after applying logistic regression before training

\begin{tabular}{llll}
\hline Variable & $\chi^{2}$ & $\mathrm{P}$ & OR \\
\hline Age & 4.544 & 0.033 & 0.987 \\
Inpatient (outpatient) & 4.045 & 0.044 & 0.070 \\
Medical (surgical) patient & 0.477 & 0.490 & 1.760 \\
CPR & 6.161 & 0.554 & 7.053 \\
Shifted to ICU & 0.350 & 0.010 & 1.481 \\
Time of arrival & 6.644 & 0.652 & 1.583 \\
During work time & 0.203 & 0.033 & 0.014 \\
Time of CPR & 4.533 & 6.152 \\
Shock & 6.080 & & 68.524 \\
\hline
\end{tabular}

CPR, cardiopulmonary resuscitation.

Table 4 The age, gender, area and cause before and after training

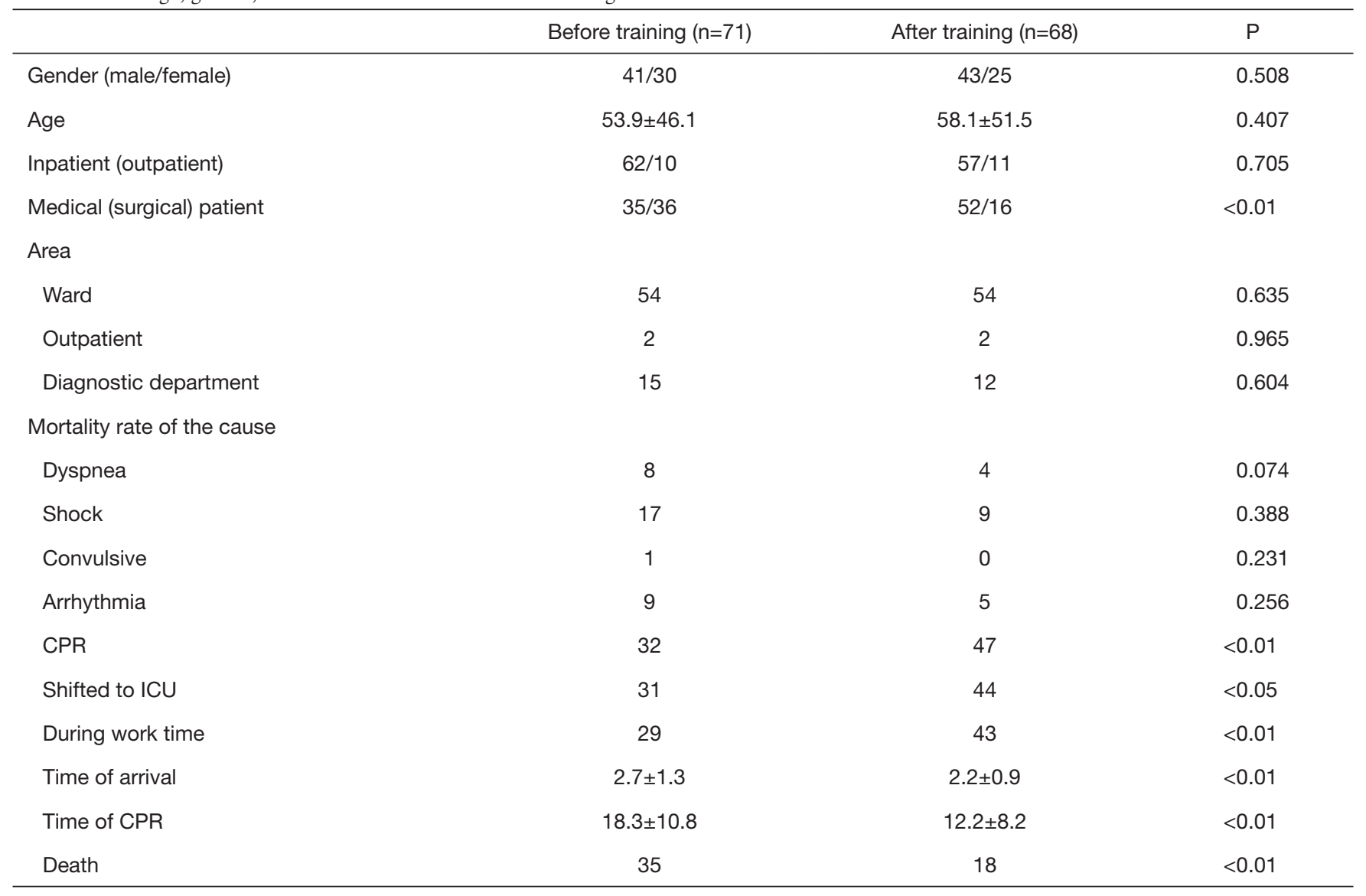

CPR, cardiopulmonary resuscitation. 
Translational Pediatrics, Vol 10, No 2 February 2021

Table 5 Survival and death group based on age, gender, area and cause before training

\begin{tabular}{|c|c|c|c|}
\hline & Death $(n=35)$ & Survived $(n=36)$ & $\mathrm{P}$ \\
\hline Age & $35.7 \pm 44.6$ & $71.6 \pm 64.6$ & $<0.05$ \\
\hline Inpatient (outpatient) & $34 / 1$ & $28 / 8$ & $<0.05$ \\
\hline Medical (surgical) patient & $12 / 23$ & $23 / 13$ & $<0.05$ \\
\hline Ward & 30 & 24 & 0.06 \\
\hline Outpatient & 1 & 1 & 0.984 \\
\hline Diagnostic department & 4 & 11 & $<0.05$ \\
\hline \multicolumn{4}{|l|}{ Cause } \\
\hline Unconsciousness & 1 & 5 & 0.095 \\
\hline Arrhythmia & 9 & 6 & 0.350 \\
\hline CPR & 35 & 18 & $<0.01$ \\
\hline Time of CPR & $24.7 \pm 9.2$ & $12.1 \pm 8.4$ & $<0.01$ \\
\hline Shifted to ICU & 35 & 16 & $<0.01$ \\
\hline During work time & 19 & 24 & 0.286 \\
\hline Time of arrival & $3.1 \pm 1.3$ & $2.3 \pm 1.2$ & $<0.05$ \\
\hline
\end{tabular}

CPR, cardiopulmonary resuscitation.

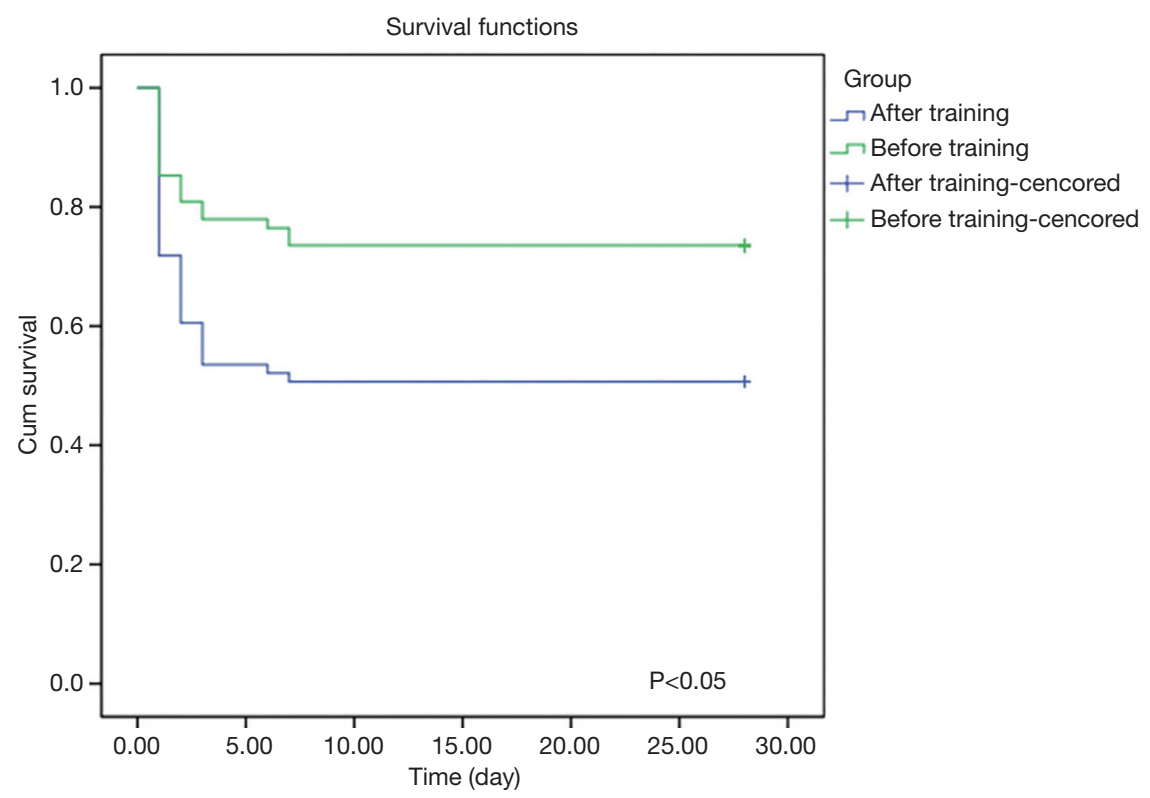

Figure 1 The survival curve before and after training. 
and consistent theoretical knowledge and establish a good basis for operation. Skill training and simulated cases closely follow the clinical reality, creating a real patientoriented clinical scene for medical staff, so that trainees can follow the rules when they encounter actual cases. We found that the time of arrival, the time of CPR, and the cause of shock were all risk factors for death in children. Kubra et al. found than the arrival times of the Code Blue teams of children's hospital ranged from 1 to 5 minutes, which was concordant with the standards, and the mean was 1.57 minutes (2). For the arrival time evaluation, the arrival times of Code Blue teams in our hospital ranged from 1 to 5 minutes, which was concordant with the standards; the mean was $2.3 \pm 1.2$ minutes in the survived group, less than the death group before training. After the training, the arrival time of the Code Blue teams shortened to $2.2 \pm$ 0.9 minutes. It is clear that the awareness of the Code Blue was improved after education of the hospital staff in our hospital. Education decreased the arrival time of the Code Blue team dramatically $(12,13)$. We also found that after training, the recovery time was significantly reduced. The proportion of patients shifted to the ICU has increased and the proportion of deaths has decreased. It was gratifying that the survival curve improved significantly after training. These changes may be due to improvements in the rescue abilities of the Code Blue team.

Since Code Blue activations do not occur frequently, the study sample was not large, and it is susceptible to children's primary diseases. The PIM or PRISM score is most commonly used to analyze the severity of illness score in pediatric patients (14). They were not calculated in this study because most of the Code Blues were too rushed and not scored and needed to be improved in the following research. For ethical reasons, historical cases are used for comparison, and the Code Blues may be affected by rising factors at the medical level.

\section{Acknowledgments}

We express our deep gratitude to the patients and the families for their willingness and cooperation in the study. Funding: None.

\section{Footnote}

Reporting Checklist: The authors have completed the STROBE reporting checklist. Available at http://dx.doi. org/10.21037/tp-20-171
Data Sharing Statement: Available at http://dx.doi. org/10.21037/tp-20-171

Peer Review File: Available at http://dx.doi.org/10.21037/tp20-171

Conflicts of Interest: All authors have completed the ICMJE uniform disclosure form (available at: Available at http:// dx.doi.org/10.21037/tp-20-171). The authors have no conflicts of interest to declare.

Ethical Statement: The authors are accountable for all aspects of the work in ensuring that questions related to the accuracy or integrity of any part of the work are appropriately investigated and resolved. This study passed the ethical review of the unit ethics committee (No. [2020]460) and followed the Helsinki Declaration (as revised in 2013). Because of the retrospective nature of the study, the requirement for informed consent was waived.

Open Access Statement: This is an Open Access article distributed in accordance with the Creative Commons Attribution-NonCommercial-NoDerivs 4.0 International License (CC BY-NC-ND 4.0), which permits the noncommercial replication and distribution of the article with the strict proviso that no changes or edits are made and the original work is properly cited (including links to both the formal publication through the relevant DOI and the license). See: https://creativecommons.org/licenses/by-nc-nd/4.0/.

\section{References}

1. Eroglu SE, Onur O, Urgan O, et al. Blue code: Is it a real emergency? World J Emerg Med 2014;5:20-3.

2. Sahin KE, Ozdinc OZ, Yoldas S, et al. Code Blue evaluation in children's hospital. World J Emerg Med 2016;7:208-12.

3. Al-Aboud KM, Al-Aboud DM. Hospital emergency codes. An appraisal. Saudi Med J 2010;31:1377.

4. Barbetti J, Lee G. Medical emergency team: a review of the literature. Nurs Crit Care 2008;13:80-5.

5. Sandroni C, Nolan J, Cavallaro F, et al. In-hospital cardiac arrest: incidence, prognosis and possible measures to improve survival. Intensive Care Med 2007;33:237-45.

6. Abe T, Tokuda Y, Ishimatsu S, et al. Predictors for good cerebral performance among adult survivors of out-ofhospital cardiac arrest. Resuscitation 2009;80:431-6.

7. Wallace DJ, Coppler P, Callaway C, et al. Selection bias, 
interventions and outcomes for survivors of cardiac arrest. Heart 2018;104:1356-61.

8. Benditt DG, Goldstein M, Sutton R, et al. Dispatcherdirected bystander initiated cardiopulmonary resuscitation: a safe step, but only a first step, in an integrated approach to improving sudden cardiac arrest survival. Circulation 2010;121:10-3.

9. de Caen AR, Berg MD, Chameides L, et al. Part 12: Pediatric Advanced Life Support: 2015 American Heart Association Guidelines Update for Cardiopulmonary Resuscitation and Emergency Cardiovascular Care. Circulation 2015;132:S526-42.

10. Porter JE, Peck B, McNabb TJ, et al. A review of Code Blue activations in a single Regional Australian Healthcare Service: A retrospective descriptive study of RISKMAN data. J Clin Nurs 2020;29:221-7.

Cite this article as: Shi Y, Liu G, Cao D, Lu G, Yuan L, Qian Y, Xu J, Sun C, Ge M, Lai L, Wang X, Lu Y, Huang $\mathrm{G}$, Zhai X. Improvement of the functioning and efficiency of a Code Blue system after training in a children's hospital in China. Transl Pediatr 2021;10(2):236-243. doi: 10.21037/tp-20-171
11. Bakan N, Karaören G, Tomruk ŞG, et al. Mortality in Code Blue; can APACHE II and PRISM scores be used as markers for prognostication?. Ulus Travma Acil Cerrahi Derg 2018;24:149-55.

12. Kinney KG, Boyd SY, Simpson DE. Guidelines for appropriate in-hospital emergency team time management: the Brooke Army Medical Center approach. Resuscitation 2004;60:33-8.

13. Hunt EA, Walker AR, Shaffner DH, et al. Simulation of in-hospital pediatric medical emergencies and cardiopulmonary arrests: highlighting the importance of the first 5 minutes. Pediatrics 2008;121:e34-43.

14. Gorgis N, Asselin JM, Fontana C, et al. Evaluation of the Association of Early Elevated Lactate With Outcomes in Children With Severe Sepsis or Septic Shock. Pediatr Emerg Care 2019;35:661-5. 